\title{
Radiative decays of the p-wave charmed heavy baryons
}

\author{
T. M. Aliev ${ }^{1, \mathrm{a}}$, T. Barakat $^{2, \mathrm{~b}}$, M. Savci ${ }^{1, \mathrm{c}}$ \\ ${ }_{1}^{1}$ Physics Department, Middle East Technical University, 06531 Ankara, Turkey \\ 2 Physics Department, King Saud University, Riyadh 11451, Saudi Arabia
}

Received: 17 March 2019 / Accepted: 11 May 2019 / Published online: 23 May 2019

(C) The Author(s) 2019

\begin{abstract}
The radiative decays of the p-wave charmed heavy baryons to the ground state baryon states are studied in the framework of the light cone QCD sum rules method. Firstly, the transition form factors that describe these transitions are estimated and then, using these form factors, the corresponding decay widths are calculated. A comparison of our results for the decay widths with those predicted by the other approaches existing in the literature is performed.
\end{abstract}

\section{Introduction}

Last 15 years are characterized by impressive progress in hadron physics experiments, especially as regards charmed hadron physics. Many new particles have been discovered [1-5]. Part of the new meson states could not be described by the conventional $(\bar{q} q)$ picture. Initiated by these observations a remarkable amount of theoretical work appeared which led to the conclusion that these states could be interpreted as molecules [6-10], tetraquarks [11,12] or hybrids [13]. A similar situation exists in the charmed baryon sector. Some of the newly discovered charmed baryon states [14-17], like the $J^{P}=\left(\frac{1}{2}\right)^{-} \Lambda_{c}$ (2595) or the $J^{P}=\left(\frac{3}{2}\right)^{-} \Lambda_{c}$ (2625), can be interpreted as meson-baryon molecules. At present, many negative parity baryons are being discovered in the charmed sector, while only the $\Sigma_{b}$ baryon has been observed in the beauty sector.

The study of the modes of the newly discovered negative parity heavy baryons can be useful for establishing their nature, i.e., the conventional $q q Q$ structure, or molecular picture, or more exotic structure. The radiative decays of these hadrons into their ground states may constitute a significant part of the total width, if the hadronic widths are suppressed by the phase volume or if the coupling constants are small.

\footnotetext{
a e-mail: taliev@metu.edu.tr

b e-mail: tbarakat@ksu.edu.sa

ce-mail: savci@metu.edu.tr
}

The radiative decays can also be useful in the determination of the quantum numbers of the negative parity states, as well as for understanding their internal structures.

The present work is devoted to the study of the radiative decays of the negative parity heavy baryons to the ground state heavy ones in the framework of the light cone QCD sum rule method (LCSR). In our analysis we have used the background field formalism, which was introduced in [18] to calculate the $\Sigma \rightarrow p \gamma$ decay. Note that the radiative decays between negative (positive) parity heavy baryons are studied in the same framework in $[19,20]$. It should also be emphasized that the decays of the heavy hadrons are studied in the framework of the LCSR in the leading order of the heavy quark effective theory [21].

\section{Transition form factors of the $B_{i} \rightarrow B_{f} \gamma$ decays at $Q^{2}=0$}

In the present section we will examine the transition form factors of the $B_{i} \rightarrow B_{f} \gamma$ decays at $Q^{2}=0$ (for the real photon). Before giving the details of the calculations, we first present the definition of the transition matrix element between the initial $\left(B_{i}\right)$ and final $\left(B_{f}\right)$ baryons states induced by the electromagnetic current, which can be written as

$$
\begin{gathered}
\left\langle B_{f}(p, s)\left|j_{\mu}\right| B_{i}\left(p^{\prime}, s\right)\right\rangle=\bar{u}^{(s)}(p)\left[\left(\gamma_{\mu}-\frac{\phi q_{\mu}}{q^{2}}\right) F_{1}\left(Q^{2}\right)\right. \\
\left.-\frac{i \sigma_{\mu \nu} q^{\nu}}{m_{i}+m_{f}} F_{2}\left(Q^{2}\right)\right] u^{\left(s^{\prime}\right)}\left(p^{\prime}\right),
\end{gathered}
$$

where $F_{1}$ and $F_{2}$ are the Dirac and the Pauli form factors, and $q=p_{1}-p_{f}$. Using this matrix element for the $B_{1} \rightarrow$ $B_{2} \gamma$ decay, we can easily calculate the decay width whose expression is

$\Gamma\left(B_{1} \rightarrow B_{2} \gamma\right)=\frac{4 \alpha|\vec{q}|^{3}}{\left(m_{1}+m_{2}\right)^{2}}\left|F_{2}(0)\right|^{2}$, 
Table 1 Light quark contents of the heavy baryons belonging to the sextet and antitriplet representations

\begin{tabular}{llllllllll}
\hline & $\Sigma_{c(b)}^{(++)+}$ & $\Sigma_{c(b)}^{+(0)}$ & $\Sigma_{c(b)}^{0(-)}$ & $\Xi_{c(b)}^{\prime 0(-)}$ & $\Xi_{c(b)}^{\prime+(0)}$ & $\Omega_{c(b)}^{0(-)}$ & $\Lambda_{c(b)}^{+(0)}$ & $\Xi_{c(b)}^{0(-)}$ & $\Xi_{c(b)}^{+(0)}$ \\
\hline$q_{1}$ & $u$ & $u$ & $d$ & $d$ & $u$ & $s$ & $u$ & $d$ & $u$ \\
$q_{2}$ & $u$ & $d$ & $d$ & $s$ & $s$ & $s$ & $d$ & $s$ & $s$ \\
\hline
\end{tabular}

where

$|\vec{q}|=\frac{m_{1}^{2}-m_{2}^{2}}{2 m_{1}}$

is the magnitude of the photon three-momentum in the rest frame of the initial baryon, and $\alpha$ is the fine structure constant.

It follows from Eq. (2) that, for an estimation of the radiative decay width, the value of the form factor $F_{2}\left(Q^{2}=0\right)$ is needed. The process $B_{i} \rightarrow B_{f} \gamma$ can be described by the correlation function

$$
\begin{gathered}
\Pi_{\mu}(p, q)=-\int \mathrm{d}^{4} x \int \mathrm{d}^{4} y \mathrm{e}^{i(p x+q y)} \\
\left\langle 0\left|\mathrm{~T}\left\{\eta_{Q_{1}}(x) J_{\mu}^{e l}(y) \bar{\eta}_{Q_{2}}(0)\right\}\right| 0\right\rangle,
\end{gathered}
$$

where $\eta_{1}$ and $\eta_{2}$ are the interpolating currents of the initial and final heavy baryons, $j_{\mu}^{e l}=e_{q} \bar{q} \gamma_{\mu} q+e_{Q} \bar{Q} \gamma_{\mu} Q$ is the electromagnetic current, $e_{q}$ and $e_{Q}$ are the electric charges for the light and heavy quarks, respectively.

According to the SU (3) classification the heavy baryons belong to the symmetric sextet and antitriplet representations with respect to the light quark exchanges. Interpolating currents corresponding to these representations are constructed in [21], which are given as

$$
\begin{aligned}
\eta_{Q}^{(S)}= & -\frac{1}{\sqrt{2}} \epsilon^{a b c}\left\{\left(q_{1}^{a T} C Q^{b}\right) \gamma_{5} q_{2}^{c}-\left(Q^{a T} C q_{2}^{b}\right) \gamma_{5} q_{1}^{c}\right. \\
& \left.+\beta\left[\left(q_{1}^{a T} C \gamma_{5} Q^{b}\right) q_{2}^{c}-\left(Q^{a T} C \gamma_{5} q_{2}^{b}\right) q_{1}^{c}\right]\right\}, \\
\eta_{Q}^{(A)}= & \frac{1}{\sqrt{6}} \epsilon^{a b c}\left\{2\left(q_{1}^{a T} C q_{2}^{b}\right) \gamma_{5} Q^{c}+\left(q_{1}^{a T} C Q^{b}\right) \gamma_{5} q_{2}^{c}\right. \\
& +\left(Q^{a T} C q_{2}^{b}\right) \gamma_{5} q_{1}^{c} \\
& +\beta\left[2 C\left(q_{1}^{a T} C \gamma_{5} q_{2}^{b}\right) Q^{c}+\left(q_{1}^{a T} C \gamma_{5} Q^{b}\right) q_{2}^{c}\right. \\
& \left.\left.+\left(Q^{a T} C \gamma_{5} q_{2}^{b}\right) q_{1}^{c}\right]\right\},
\end{aligned}
$$

where $a, b, c$ are the color indices, and $\beta$ is the arbitrary auxiliary parameter. The light quark contents of the heavy baryons in the 6 and $\overline{3}$ representations are given in Table 1 .

The decay constant of the baryon interpolating current is defined by

$\left\langle 0\left|\eta_{Q_{i}}\right| B(p, s)\right\rangle=\lambda_{i} u(p)$.

Introducing an electromagnetic background field of the plane wave $F_{\mu \nu}=i\left(\varepsilon_{\nu}^{(\lambda)} q_{\mu}-\varepsilon_{\mu}^{(\lambda)} q_{\nu}\right) \mathrm{e}^{i q x}$, the correlation function given in Eq. (3) can be rewritten as

$$
\Pi_{\mu}(p, q) \epsilon^{\mu}=i \int \mathrm{d}^{4} x e^{i q x}\left\langle 0\left|\mathrm{~T}\left\{\eta_{Q_{1}}(x) \bar{\eta}_{Q_{2}}(0)\right\}\right| 0\right\rangle_{F},
$$

where the subscript $F$ means that all calculations are performed in the background field $F_{\mu \nu}$. The background field method was introduced in [22] in order to calculate the decay width of the $\Sigma \rightarrow p \gamma$ process.

Let us now consider the transitions involving heavy hadrons. Along the lines suggested by the usual sum rules method, we insert a complete set of heavy baryons between the interpolating currents $\eta_{1}$ and $\eta_{2}$, and the electromagnetic current in the correlation function given in Eq. (3). In doing so, there appears a problem which is absent in the case of mesons, namely: the chosen form of the interpolating current couples not only with the positive parity ground state baryons $J^{P}=\left(\frac{1}{2}\right)^{+}$but also with a heavier baryon resonance with the negative parity $J^{P}=\left(\frac{1}{2}\right)^{-}$. Many of the heavy baryons, especially the ones involving the $c$-quark, have already been observed in the experiments. Hereafter, the negative (positive) parity baryons and their masses will be denoted as $B^{*}(B)$ and $m_{B^{*}}\left(m_{B}\right)$ and their masses will be denoted as $B^{*}$ and $m_{B^{*}}$, respectively.

The mass difference between the negative parity and ground state positive parity baryons is about $300 \mathrm{MeV}$, and a similar mass difference is expected for the baryons with the $b$-quark. Consequently, we should take into account the contribution of the negative parity baryons in the correlation function. Keeping these remarks in mind, from the hadronic side, the correlation function containing double poles can be written as

$$
\begin{aligned}
& \Pi_{\mu}(p, q) \varepsilon^{\mu}=\varepsilon^{\mu} \sum_{\alpha, \beta} \frac{\left\langle 0\left|\eta_{Q_{2}}\right| B_{2 \alpha}(p, s)\right\rangle}{p^{2}-m_{2 \alpha}^{2}} \\
& \quad\left\langle B_{2 \alpha}(p, s)\left|j_{\mu}^{e l}\right| B_{1 \beta}\right\rangle \frac{\left\langle B_{1 \beta}(p+q, s)\left|\bar{\eta}_{Q_{1}}\right| 0\right\rangle}{(p+q)^{2}-m_{1 \beta}^{2}}+\cdots
\end{aligned}
$$

where summation over $\alpha$ and $\beta$ is performed over the positive and the negative parity baryons, and dots indicate the contributions of higher states and of the continuum. The matrix elements in Eq. (6) are defined as

$$
\begin{aligned}
& \left\langle 0\left|\eta_{Q}\right| B_{2}(p, s)\right\rangle=\lambda_{2} u_{2}(p, s), \\
& \quad\left\langle 0\left|\eta_{Q}\right| B_{2}^{*}(p, s)\right\rangle=\lambda_{2}^{*} \gamma_{5} u_{2}^{*}(p, s), \\
& \left\langle B_{2}(p, s)\left|j_{\mu}^{e l}\right| B_{1}(p+q, s)\right\rangle=\bar{u}_{2}\left[\left(\gamma_{\mu}-\frac{q q_{\mu}}{q^{2}}\right) F_{1}\right. \\
& \left.\quad-\frac{i \sigma_{\mu \nu} q^{\nu}}{m_{1}+m_{2}} F_{2}\right] u_{1}(p+q, s)
\end{aligned}
$$


where $\lambda_{2} \lambda_{2}^{*}$ are the residues of the baryons $B_{2}$ and $B_{2}^{*}$, and $F_{1}$ and $F_{2}$ are the form factors for the corresponding transitions, respectively.

The matrix elements $\left\langle B_{2}^{*}\left|j_{\mu}^{e l}\right| B_{1}\right\rangle,\left\langle B_{2}\left|j_{\mu}^{e l}\right| B_{1}^{*}\right\rangle$, and $\left\langle B_{2}^{*}\left|j_{\mu}^{e l}\right| B_{1}^{*}\right\rangle$ can easily be obtained from Eq. (8) with the help of the replacements $\bar{u}_{2} \rightarrow \bar{u}_{2}^{*} \gamma_{5}, m_{2} \rightarrow m_{2}^{*}, u_{1} \rightarrow \gamma_{5} u_{1}$, and $\bar{u}_{2} \rightarrow \bar{u}_{2}^{*} \gamma_{5}, u_{1} \rightarrow \gamma_{5} u_{1}^{*}, m_{1} \rightarrow m_{1}^{*}, m_{2} \rightarrow m_{2}^{*}$, respectively. As we already noted that the conservation of the electromagnetic current leads to the result that the radiative decay is described only by the form factor $F_{2}$.

Using the relation

$\sum_{s} u(p)^{(s)} \bar{u}(p)^{(s)}=\not p+m$,

for the summation of the Dirac spinors over the spin, and using the transversality condition $q \cdot \varepsilon=0$ for the photon field, one can easily show that only the $(p \cdot \varepsilon)$ term is proportional to $F_{2}$.

Separating the terms proportional to $F_{2-+}$, we get the following result for the correlation function from the phenomenological part:

$$
\begin{aligned}
\Pi_{\mu} \varepsilon^{\mu}= & -A_{++}\left(p+m_{2}\right)\left(p+\not d+m_{1}\right)(p \cdot \varepsilon) \\
& +A_{--}\left(p-m_{2^{*}}\right)\left(p-\not d-m_{1^{*}}\right)(p \cdot \varepsilon) \\
& +A_{+-}\left(\not b-m_{2^{*}}\right)\left(p+q+m_{1}\right)(p \cdot \varepsilon) \\
& -A_{-+}\left(p+m_{2}\right)\left(p+\not d-m_{1^{*}}\right)(p \cdot \varepsilon)
\end{aligned}
$$

where

$A_{i j}=\frac{2 \lambda_{1^{i}} \lambda_{2^{j}}}{\left(p^{2}-m_{2^{j}}^{2}\right)\left[(p+q)^{2}-m_{1^{i}}^{2}\right]} \frac{F_{2 i j}}{\left(m_{1^{i}}+m_{2^{j}}\right)}$.

As has already been noted, in this work we study the radiative decays of the transitions of the negative parity heavy baryons to positive heavy baryons. The invariant function $A_{-+}$in Eq. (9) describes these radiative decays under consideration. Therefore the other invariant functions should be eliminated. It follows from Eq. (9) that we have four independent invariant functions, and hence we need four equations in order to solve for the invariant function $A_{-+}$. The four equations can be obtained from the coefficients of the structures $(p \cdot \varepsilon) \not p d,(p \cdot \varepsilon) p,(p \cdot \varepsilon) d$, and $(p \cdot \varepsilon) I$.

In order to obtain the sum rules for the form factors $F_{2-+}(0)$ the expression of the correlation function from the QCD side is needed. This correlation function can be calculated using the operator product expansion (OPE). The OPE is performed in the deep Euclidean region for the variables $p^{2},(p+q)^{2} \ll m_{Q}^{2}$. In the LCSR method, the OPE is performed over the twists of the nonlocal operators. The expansion of the nonlocal operators up to twist- 4 is performed in [18], where the four-particle contributions are neglected. Following this work we also neglect them. The matrix elements of the nonlocal operators between the vacuum and the photon state are parametrized in terms of the photon distribution amplitudes (DAs). The photon DAs are studied comprehensively in [23], and therefore we do not present them in this work.

Equating the coefficients of the structures $(p \cdot \varepsilon) \not p d,(p \cdot \varepsilon) \not p$, $(p \cdot \varepsilon) d,(p \cdot \varepsilon) I$ from the OPE side to the double hadronic dispersion relations, we obtain the following four linearly independent equations from which we can solve for the form factor $F_{2-+}$ :

$$
\begin{aligned}
& -A_{++}+A_{--}+A_{+-}-A_{-+}=\Pi_{1}, \\
& -\left(m_{1}+m_{2}\right) A_{++}-\left(m_{1^{*}}+m_{2^{*}}\right) A_{--} \\
& +\left(m_{1}-m_{2^{*}}\right) A_{+-}-\left(m_{1^{*}}-m_{2}\right) A_{-+}=\Pi_{2}, \\
& -m_{2} A_{++}-m_{2^{*}} A_{--}-m_{2^{*}} A_{+-}-m_{2} A_{-+}=\Pi_{3}, \\
& -m_{2}\left(m_{1}+m_{2}\right) A_{++}+m_{2^{*}}\left(m_{1^{*}}+m_{2^{*}}\right) A_{--} \\
& -m_{2^{*}}\left(m_{1}-m_{2^{*}}\right) A_{+-}+m_{2}\left(m_{1^{*}}-m_{2}\right) A_{-+}=\Pi_{4} .
\end{aligned}
$$

Solving the set of four equations in Eq. (11), we can easily find $A_{-+}$. Performing the double Borel transformation over the variables $(p+q)^{2} \rightarrow M_{1}^{2}, p^{2} \rightarrow M_{2}^{2}$, we obtain the LCSR for the form factor $F_{2-+}$ at the point $Q^{2}=0$ whose expression is given as

$$
\begin{aligned}
& F_{2-+}\left(Q^{2}=0\right)=\frac{m_{1^{*}}+m_{2}}{2 \lambda_{1 *} \lambda_{2}} \mathrm{e}^{-m_{1^{-}}^{2} / M_{1}^{2}-m_{2^{-}}^{2} / M_{2}^{2}} \\
& \quad\left\{\frac{1}{\left(m_{1^{*}}+m_{1}\right)\left(m_{2^{*}}+m_{2}\right)}\right. \\
& \quad \times\left[m_{2^{*}}\left(m_{1}+m_{2}\right) \Pi_{1}-m_{2^{*}} \Pi_{2}\right. \\
& \left.\left.+\left(m_{1}+m_{2}\right) \Pi_{3}-\Pi_{4}\right]\right\} \\
& +\int \mathrm{d} s_{1} \mathrm{~d} s_{2} \rho^{h}\left(s_{1}, s_{2}\right) \mathrm{e}^{-s_{1} / M_{1}^{2}-s_{2} / M_{2}^{2}}
\end{aligned}
$$

where $\Pi_{i}^{B}$ denote the Borel-transformed invariant functions in the coefficients of the aforementioned Lorentz structures. The expressions of the invariant functions $\Pi_{i}^{B}$ are quite lengthy and therefore we do not present them in this work. The function $\rho^{h}\left(s_{1}, s_{2}\right)$ in the last term is the hadronic spectral density of all excited and continuum states with the quantum numbers of $B^{*}$. The hadronic spectral density is estimated by using the quark-hadron duality ansatz. The residues of the corresponding positive (negative) parity baryons are calculated in [24-26]. In our calculations for residues, we have used the results of this work.

For the processes under consideration, we choose $M_{1}^{2}=$ $M_{2}^{2}=2 M^{2}$ since the masses of the initial and final baryons are quite close to each other. The continuum subtraction can be carried out with the help of the transformation

$$
\left(M^{2}\right)^{n} \mathrm{e}^{-m_{Q}^{2} / M^{2}} \rightarrow \frac{1}{\Gamma(n)} \int_{m_{Q}^{2}}^{s_{0}} \mathrm{~d} s\left(s-m^{2}\right)^{n-1} \mathrm{e}^{-s / M^{2}} \quad(n \geq 1) .
$$


For the terms that are proportional to the zeroth or negative power of $M^{2}$ or negative powers of $M^{2}$ the continuum subtraction is not performed because these contributions are negligibly small (see for details [27]).

\section{Numerical analysis}

In order to perform the numerical analysis for the form factor $F_{2-+}(0)$, we first introduce the input parameters which we shall use in further calculations. The main ingredient in any of the LCSR approaches in calculating the form factors is the set of photon DAs of the particle under consideration (in our case photon DAs). The photon DAs are obtained in [23], and for completeness, we present their expressions in Appendix A. The mass of the negative and positive parity baryons are taken from [29]. The mass of the virtual $c$ quark appearing in the heavy quark propagator is set to its value calculated in the $\overline{\mathrm{MS}}$ scheme which is given as $\bar{m}_{c}\left(\bar{m}_{c}\right)=1.28 \pm 0.03 \mathrm{GeV}$ [26]. In our analysis for the values of the quark condensates we use $\langle\bar{q} q\rangle(1 \mathrm{GeV})=-\left(246_{-19}^{+28} \mathrm{MeV}\right)^{3}[30,31],\langle\bar{s} s\rangle(1 \mathrm{GeV})=$ $0.8\langle\bar{q} q\rangle(1 \mathrm{GeV}), m_{0}^{2}=(0.8 \pm 0.2) \mathrm{GeV}^{2}$ [32]. The value of the magnetic susceptibility we have used is $\chi(1 \mathrm{GeV})=$ $-0.285 \mathrm{GeV}^{-2}$ [33].

The sum rule for the form factors $F_{2-+}(0)$, in addition to the aforementioned input parameters, contain also three auxiliary parameters. These parameters are the Borel mass parameter $M^{2}$, the continuum threshold $s_{0}$, and the arbitrary parameter $\beta$ appearing in the expressions of the interpolating currents. According to the QCD sum rules methodology we need to find the so-called working regions of these parameters, where $F_{2-+}(0)$ are insensitive to the variation of these parameters in their working regions. We use the standard criteria of the sum rules, which requires that both power corrections and continuum contributions should sufficiently be suppressed. The result of the analysis of the sum rules for the negative parity heavy baryons, which has been studied in [31], leads to the following intervals for the Borel parameter $M^{2}$ :

$$
\begin{aligned}
& 2.5 \mathrm{GeV}^{2} \leq M^{2} \leq 4.0 \mathrm{GeV}^{2}, \text { for } \Sigma_{c}, \Xi_{c}^{\prime}, \Lambda_{c}, \Xi_{c}, \\
& 4.5 \mathrm{GeV}^{2} \leq M^{2} \leq 7.0 \mathrm{GeV}^{2} \text {, for } \Sigma_{b}, \Xi_{b}^{\prime}, \Lambda_{b}, \Xi_{b} \text {. }
\end{aligned}
$$

The range of the values of the continuum threshold $s_{0}$ is determined by imposing the condition that the sum rules should reproduce the measured values of the form factors to within $10-15 \%$ accuracy, from which we obtain $11 \mathrm{GeV}^{2} \leq s_{0} \leq$ $14.0 \mathrm{GeV}^{2}$.

Our final attempt is focused on the determination of the working region of the arbitrary parameter $\beta$. The working domain of the parameter $\beta$ is obtained by demanding that the form factor $F_{2-+}(0)$ shows good stability with respect to the variation of $\beta$. We have gone through an analysis for all possible transitions, and the form factors $F_{2-+}(0)$ seem to be practically insensitive to the variation in $\cos \theta$ in the domain $-1 \leq \cos \theta \leq-0.5$; we observed that this working region of $\cos \theta$ is practically common for all transitions. Our final results for the form factor $F_{2-+}(0)$ for all transition channels are given by

$$
F_{2-+}(0)= \begin{cases}(0.5 \pm 0.05) & \Sigma_{c}^{0 *}(2792) \rightarrow \Sigma_{c}^{0} \gamma \\ (-1.0 \pm 0.2) & \Sigma_{c}^{+*}(2792) \rightarrow \Sigma_{c}^{+} \gamma \\ (-3.5 \pm 0.05) & \Sigma_{c}^{++*}(2792) \rightarrow \Sigma_{c}^{++} \gamma \\ (2.5 \pm 0.5) & \Xi_{c}^{\prime 0 *}(2870) \rightarrow \Xi_{c}^{\prime 0} \gamma \\ (-1 \pm 0.1) & \Xi_{c}^{\prime+*}(2870) \rightarrow \Xi_{c}^{\prime+} \gamma \\ (2.5 \pm 0.5) & \Sigma_{c}^{+*}(2792) \rightarrow \Lambda_{c}^{+} \gamma \\ (-0.3 \pm 0.05) & \Xi_{c}^{\prime 0 *}(2870) \rightarrow \Xi_{c}^{0} \gamma \\ (2 \pm 0.3) & \Xi_{c}^{\prime+*}(2870) \rightarrow \Xi_{c}^{+} \gamma \\ (0.3 \pm 0.07) & \Xi_{c}^{0 *}(2790) \rightarrow \Xi_{c}^{0} \gamma \\ (3 \pm 0.5) & \Xi_{c}^{+*}(2790) \rightarrow \Xi_{c}^{+} \gamma \\ (2.5 \pm 0.4) & \Lambda_{c}^{+*}(2592) \rightarrow \Lambda_{c}^{+} \gamma \\ (3 \pm 0.5) & \Lambda_{c}^{+*}(2592) \rightarrow \Sigma_{c}^{+} \gamma \\ (0.25 \pm 0.05) & \Xi_{c}^{0 *}(2790) \rightarrow \Xi_{c}^{\prime 0} \gamma \\ (2.5 \pm 0.5) & \Xi_{c}^{+*}(2790) \rightarrow \Xi_{c}^{\prime+} \gamma\end{cases}
$$

The errors in the results are estimated by changing various input parameters within their working regions, and by also taking into consideration the resulting separate errors of the form factors $F_{2-+}(0)$ quadratically.

Having obtained the values of the form factors $F_{2-+}(0)$, we can calculate the decay widths of the decays under consideration. The values of the decay widths are presented in Table 2. For completeness we also present the values of the decay widths for the same transitions obtained in the framework of the other approaches such as the coupled channel dynamically generated model [34], relativistic quark model [35], the light cone sum rule method [36], chiral perturbation theory [37], the bound state picture [38], and the constituent quark model [39].

Reviewing the values of the decay widths given in Table 1, we see that between our results and predictions coming from other approaches on many channels, there are considerable differences. Therefore, measurement of decay widths can serve as a useful tool for "choosing the right model". From Table 1, we conclude that our approach predicts quite large values, especially for the decay channels, and these channels $\left(\Sigma_{c}^{++*} \rightarrow \Sigma_{c}^{++} \gamma, \Xi_{c}^{\prime 0 *} \rightarrow \Xi_{c}^{\prime 0} \gamma, \Sigma_{c}^{+*} \rightarrow \Lambda_{c}^{+} \gamma\right.$, $\Xi_{c}^{\prime * *} \rightarrow \Xi_{c}^{+} \gamma, \Xi_{c}^{+*} \rightarrow \Xi_{c}^{+} \gamma$, and $\left.\Lambda_{c}^{+*} \rightarrow \Lambda_{c}^{+} \gamma\right)$ can be observed in the new future in experiments conducted at accelerators. Here we would like to note that part of the considered decays within the same framework have been studied in [36] and we see that there are essential differences between our predictions and the ones obtained in [36].

These differences can be explained as follows. In [36] all calculations is performed in the framework of HQET. It is known that the HQET works very well for the systems involving the b-quark. However, it is also known that $1 / m_{Q}$ 
Table 2 The values of radiative decay widths (in units of $\mathrm{keV}$ )

\begin{tabular}{lllllllll}
\hline & This work & {$[34]$} & {$[35]$} & {$[36]$} & {$[37]$} & {$[38]$} & {$[39]$} & \\
\hline$\Sigma_{c}^{0 *}(2792) \rightarrow \Sigma_{c}^{0} \gamma$ & $9(1 \pm 0.2)$ & 9 & - & - & - & - & 5.02 & - \\
$\Sigma_{c}^{+*}(2792) \rightarrow \Sigma_{c}^{+} \gamma$ & $36(1 \pm 0.4)$ & 29 & - & - & - & - & 0.92 & - \\
$\Sigma_{c}^{++*}(2792) \rightarrow \Sigma_{c}^{++} \gamma$ & $440(1 \pm 0.25)$ & 51 & - & - & - & & 8.54 & - \\
$\Xi_{c}^{\prime 0 *}(2870) \rightarrow \Xi_{c}^{\prime 0} \gamma$ & $132(1 \pm 0.2)$ & & & & & & & \\
$\Xi_{c}^{\prime+*}(2870) \rightarrow \Xi_{c}^{\prime+} \gamma$ & $21(1 \pm 0.2)$ & & & & & & & \\
$\Sigma_{c}^{+*}(2792) \rightarrow \Lambda_{c}^{+} \gamma$ & $701(1 \pm 0.4)$ & 35 & - & & - & - & 52.1 & - \\
$\Xi_{c}^{\prime 0 *}(2870) \rightarrow \Xi_{c}^{0} \gamma$ & $4.8(1 \pm 0.3)$ & & & & & & & \\
$\Xi_{c}^{\prime+*}(2870) \rightarrow \Xi_{c}^{+} \gamma$ & $214(1 \pm 0.3)$ & & & & & & & \\
$\Xi_{c}^{0 *}(2790) \rightarrow \Xi_{c}^{0} \gamma$ & $2.7(1 \pm 0.3)$ & 117 & - & - & - & - & 263 & 5.57 \\
$\Xi_{c}^{+*}(2790) \rightarrow \Xi_{c}^{+} \gamma$ & $265(1 \pm 0.4)$ & 246 & - & - & - & - & 4.65 & 1.39 \\
$\Lambda_{c}^{+*}(2592) \rightarrow \Lambda_{c}^{+} \gamma$ & $189(1 \pm 0.3)$ & 278 & $115 \pm 1$ & 36 & 0 & 16 & 0.26 & 1.59 \\
$\Lambda_{c}^{+*}(2592) \rightarrow \Sigma_{c}^{+} \gamma$ & $29(1 \pm 0.3)$ & 2 & $77 \pm 1$ & 11 & 71 & - & 0.45 & 41.6 \\
$\Xi_{c}^{0 *}(2790) \rightarrow \Xi_{c}^{\prime 0} \gamma$ & $0.54(1 \pm 0.4)$ & 1 & - & - & - & - & 0 & 0 \\
$\Xi_{c}^{+*}(2790) \rightarrow \Xi_{c}^{\prime+} \gamma$ & $54(1 \pm 0.4)$ & 1 & - & - & - & - & 1.43 & 128 \\
\hline
\end{tabular}

expansion terms would give considerable contributions for systems with the c-quark. Another difference is related to the way we treat the baryon currents. On our side, both positive and negative parity baryons are taken into account. Later, with the use of the sum rules obtained from different Lorentz structures, the contributions coming from the negative (positive) to negative (positive), as well as positive to negative parity baryon transitions are all estimated. While the interaction between the baryon current and the negative/positive parity baryons is assumed to be quite general in our case, only negative to positive baryon transitions are taken into account in Ref. [36]. Finally, we have used the improved light cone photon distribution amplitudes on our work. Hence, these all add up to account for the ballpark of the differences.

Our final remark on this section is that the decay widths for the corresponding beauty baryons can be obtained from these calculations by performing the relevant replacements.

\section{Conclusion}

In this work, we study the radiative decays of the negative parity heavy baryons to the ground state positive parity heavy baryons in the framework of the LCSR method. We find that the transitions $\Sigma_{c}^{++*} \rightarrow \Sigma_{c}^{++} \gamma, \Xi_{c}^{\prime 0 *} \rightarrow \Xi_{c}^{\prime 0} \gamma, \Sigma_{c}^{+*} \rightarrow$ $\Lambda_{c}^{+} \gamma, \Xi_{c}^{\prime * *} \rightarrow \Xi_{c}^{+} \gamma, \Xi_{c}^{+*} \rightarrow \Xi_{c}^{+} \gamma$, and $\Lambda_{c}^{+*} \rightarrow \Lambda_{c}^{+} \gamma$ have sizable decay width values. Our results show that the decay widths calculated in this work are quite different from the ones predicted by the other approaches. The sizable values of the decay widths we obtain indicate that these radiative decays can serve as a handy tool for gaining information as regards the properties of the negative parity heavy baryons.
Acknowledgements One of us, T. Barakat, thanks the International Scientific Partnership Program ISPP at the King Saud University for funding his research work through ISPP No: 0038.

Data Availability Statement This manuscript has no associated data or the data will not be deposited. [Authors' comment: This is a theoretical study and no actual data has been used.]

Open Access This article is distributed under the terms of the Creative Commons Attribution 4.0 International License (http://creativecomm ons.org/licenses/by/4.0/), which permits unrestricted use, distribution, and reproduction in any medium, provided you give appropriate credit to the original author(s) and the source, provide a link to the Creative Commons license, and indicate if changes were made.

Funded by SCOAP ${ }^{3}$.

\section{Appendix A: photon distribution amplitudes (DA's)}

In the present appendix, for completeness we present the definitions of the photon DAs obtained in [23]. We have

$$
\begin{aligned}
& \left\langle\gamma(q)\left|\bar{q}(x) \sigma_{\mu \nu} q(0)\right| 0\right\rangle=-i e_{q}\langle\bar{q} q\rangle\left(\varepsilon_{\mu} q_{\nu}-\varepsilon_{\nu} q_{\mu}\right) \\
& \int_{0}^{1} \mathrm{~d} u \mathrm{e}^{i \bar{u} q x}\left(\chi \varphi_{\gamma}(u)+\frac{x^{2}}{16} \mathbb{A}(u)\right) \\
& \quad-\frac{i}{2(q x)} e_{q}\langle\bar{q} q\rangle\left[x_{\nu}\left(\varepsilon_{\mu}-q_{\mu} \frac{\varepsilon x}{q x}\right)-x_{\mu}\left(\varepsilon_{\nu}-q_{\nu} \frac{\varepsilon x}{q x}\right)\right] \\
& \quad \int_{0}^{1} \mathrm{~d} u \mathrm{e}^{i \bar{u} q x} h_{\gamma}(u) \\
& \left\langle\gamma(q)\left|\bar{q}(x) \gamma_{\mu} q(0)\right| 0\right\rangle \\
& =e_{q} f_{3 \gamma}\left(\varepsilon_{\mu}-q_{\mu} \frac{\varepsilon x}{q x}\right) \int_{0}^{1} \mathrm{~d} u \mathrm{e}^{i \bar{u} q x} \psi^{v}(u) \\
& \left\langle\gamma(q)\left|\bar{q}(x) \gamma_{\mu} \gamma_{5} q(0)\right| 0\right\rangle \\
& =-\frac{1}{4} e_{q} f_{3 \gamma} \epsilon_{\mu \nu \alpha \beta} \varepsilon^{v} q^{\alpha} x^{\beta} \int_{0}^{1} \mathrm{~d} u \mathrm{e}^{i \bar{u} q x} \psi^{a}(u)
\end{aligned}
$$




$$
\begin{aligned}
& \left\langle\gamma(q)\left|\bar{q}(x) g_{s} G_{\mu \nu}(v x) q(0)\right| 0\right\rangle \\
& =-i e_{q}\langle\bar{q} q\rangle\left(\varepsilon_{\mu} q_{\nu}-\varepsilon_{\nu} q_{\mu}\right) \int \mathcal{D} \alpha_{i} \mathrm{e}^{i\left(\alpha_{\bar{q}}+v \alpha_{g}\right) q x} \mathcal{S}\left(\alpha_{i}\right) \\
& \left\langle\gamma(q)\left|\bar{q}(x) g_{s} \widetilde{G}_{\mu \nu} i \gamma_{5}(v x) q(0)\right| 0\right\rangle \\
& =-i e_{q}\langle\bar{q} q\rangle\left(\varepsilon_{\mu} q_{\nu}-\varepsilon_{\nu} q_{\mu}\right) \int \mathcal{D} \alpha_{i} \mathrm{e}^{i\left(\alpha_{\bar{q}}+v \alpha_{g}\right) q x} \widetilde{\mathcal{S}}\left(\alpha_{i}\right) \\
& \left\langle\gamma(q)\left|\bar{q}(x) g_{s} \widetilde{G}_{\mu \nu}(v x) \gamma_{\alpha} \gamma_{5} q(0)\right| 0\right\rangle \\
& =e_{q} f_{3 \gamma} q_{\alpha}\left(\varepsilon_{\mu} q_{\nu}-\varepsilon_{\nu} q_{\mu}\right) \int \mathcal{D} \alpha_{i} \mathrm{e}^{i\left(\alpha_{\bar{q}}+v \alpha_{g}\right) q x} \mathcal{A}\left(\alpha_{i}\right) \\
& \left\langle\gamma(q)\left|\bar{q}(x) g_{s} G_{\mu \nu}(v x) i \gamma_{\alpha} q(0)\right| 0\right\rangle \\
& =e_{q} f_{3 \gamma} q_{\alpha}\left(\varepsilon_{\mu} q_{\nu}-\varepsilon_{\nu} q_{\mu}\right) \int \mathcal{D} \alpha_{i} \mathrm{e}^{i\left(\alpha_{\bar{q}}+v \alpha_{g}\right) q x} \mathcal{V}\left(\alpha_{i}\right) \\
& \left\langle\gamma(q)\left|\bar{q}(x) \sigma_{\alpha \beta} g_{s} G_{\mu \nu}(v x) q(0)\right| 0\right\rangle \\
& =e_{q}\langle\bar{q} q\rangle\left\{\left[\left(\varepsilon_{\mu}-q_{\mu} \frac{\varepsilon x}{q x}\right)\left(g_{\alpha \nu}-\frac{1}{q x}\left(q_{\alpha} x_{v}+q_{v} x_{\alpha}\right)\right)\right.\right. \\
& q \beta \\
& -\left(\varepsilon_{\mu}-q_{\mu} \frac{\varepsilon x}{q x}\right) \\
& \left(g_{\beta \nu}-\frac{1}{q x}\left(q_{\beta} x_{v}+q_{\nu} x_{\beta}\right)\right) q_{\alpha} \\
& -\left(\varepsilon_{v}-q_{v} \frac{\varepsilon x}{q x}\right) \\
& \left(g_{\alpha \mu}-\frac{1}{q x}\left(q_{\alpha} x_{\mu}+q_{\mu} x_{\alpha}\right)\right) q_{\beta} \\
& \left.+\left(\varepsilon_{v}-q_{v} \frac{\varepsilon x}{q \cdot x}\right)\left(g_{\beta \mu}-\frac{1}{q x}\left(q_{\beta} x_{\mu}+q_{\mu} x_{\beta}\right)\right) q_{\alpha}\right] \\
& \int \mathcal{D} \alpha_{i} \mathrm{e}^{i\left(\alpha_{\bar{q}}+v \alpha_{g}\right) q x} \mathcal{T}_{1}\left(\alpha_{i}\right) \\
& +\left[\left(\varepsilon_{\alpha}-q_{\alpha} \frac{\varepsilon x}{q x}\right)\right. \\
& \left(g_{\mu \beta}-\frac{1}{q x}\left(q_{\mu} x_{\beta}+q_{\beta} x_{\mu}\right)\right) q_{v} \\
& -\left(\varepsilon_{\alpha}-q_{\alpha} \frac{\varepsilon x}{q x}\right)\left(g_{\nu \beta}-\frac{1}{q x}\left(q_{\nu} x_{\beta}+q_{\beta} x_{\nu}\right)\right) q_{\mu} \\
& -\left(\varepsilon_{\beta}-q_{\beta} \frac{\varepsilon x}{q x}\right)\left(g_{\mu \alpha}-\frac{1}{q x}\left(q_{\mu} x_{\alpha}+q_{\alpha} x_{\mu}\right)\right) q_{\nu} \\
& \left.+\left(\varepsilon \beta-q_{\beta} \frac{\varepsilon x}{q x}\right)\left(g_{\nu \alpha}-\frac{1}{q x}\left(q_{\nu} x_{\alpha}+q_{\alpha} x_{\nu}\right)\right) q_{\mu}\right] \\
& \int \mathcal{D} \alpha_{i} \mathrm{e}^{i\left(\alpha_{\bar{q}}+v \alpha_{g}\right) q x} \mathcal{T}_{2}\left(\alpha_{i}\right) \\
& +\frac{1}{q x}\left(q_{\mu} x_{v}-q_{\nu} x_{\mu}\right)\left(\varepsilon_{\alpha} q_{\beta}-\varepsilon_{\beta} q_{\alpha}\right) \\
& \int \mathcal{D} \alpha_{i} \mathrm{e}^{i\left(\alpha_{\bar{q}}+v \alpha_{g}\right) q x} \mathcal{T}_{3}\left(\alpha_{i}\right) \\
& +\frac{1}{q x}\left(q_{\alpha} x_{\beta}-q_{\beta} x_{\alpha}\right)\left(\varepsilon_{\mu} q_{\nu}-\varepsilon_{\nu} q_{\mu}\right) \\
& \left.\int \mathcal{D} \alpha_{i} \mathrm{e}^{i\left(\alpha_{\bar{q}}+v \alpha_{g}\right) q x} \mathcal{T}_{4}\left(\alpha_{i}\right)\right\} \\
& \left\langle\gamma(q)\left|\bar{q}(x) e_{q} F_{\mu \nu}(v x) q(0)\right| 0\right\rangle=-i e_{q}\langle\bar{q} q\rangle\left(\varepsilon_{\mu} q_{\nu}-\varepsilon_{\nu} q_{\mu}\right) \\
& \int \mathcal{D} \alpha_{i} \mathrm{e}^{i\left(\alpha_{\bar{q}}+v \alpha_{g}\right) q x} \mathcal{S}^{\gamma}\left(\alpha_{i}\right) \\
& \left\langle\gamma(q)\left|\bar{q}(x) \sigma_{\alpha \beta} F_{\mu \nu}(v x) q(0)\right| 0\right\rangle
\end{aligned}
$$

$$
\begin{aligned}
= & e_{q}\langle\bar{q} q\rangle \frac{1}{q x}\left(q_{\alpha} x_{\beta}-q_{\beta} x_{\alpha}\right)\left(\varepsilon_{\mu} q_{\nu}-\varepsilon_{\nu} q_{\mu}\right) \\
& \times \int \mathcal{D} \alpha_{i} \mathrm{e}^{i\left(\alpha_{\bar{q}}+v \alpha_{g}\right) q x} \mathcal{T}_{4}^{\gamma}\left(\alpha_{i}\right),
\end{aligned}
$$

where $\varphi_{\gamma}(u)$ is the leading twist-2, $\psi^{v}(u), \psi^{a}(u), \mathcal{A}$ and $\mathcal{V}$ are the twist-3, and $h_{\gamma}(u), \mathbb{A}, \mathcal{S}, \widetilde{\mathcal{S}}, \mathcal{S}^{\gamma}, \mathcal{T}_{i}(i=1,2,3,4)$, $\mathcal{T}_{4}^{\gamma}$ are the twist- 4 photon DAs, $\chi$ is the magnetic susceptibility, and the measure $\mathcal{D} \alpha_{i}$ is defined as

$$
\int \mathcal{D} \alpha_{i}=\int_{0}^{1} \mathrm{~d} \alpha_{\bar{q}} \int_{0}^{1} \mathrm{~d} \alpha_{q} \int_{0}^{1} \mathrm{~d} \alpha_{g} \delta\left(1-\alpha_{\bar{q}}-\alpha_{q}-\alpha_{g}\right) .
$$

\section{References}

1. E.S. Swanson, Phys. Rep. B 429, 243 (2006)

2. S. Godfrey, S.L. Olsen, Annu. Rev. Nucl. Part. Sci. 58, 51 (2008)

3. M.B. Voloshin, Prog. Part. Nucl. Phys. 61, 455 (2008)

4. M. Nielsen, F.S. Navarra, S.H. Lee, Phys. Rep. D 497, 41 (2010)

5. H.-X. Chen, X. Liu, Y.-R. Liu, S.L. Zhu, Rep. Prog. Phys. 80, 076201 (2017)

6. T. Barnes, F.E. Close, H.J. Lipkin, Phys. Rev. D 68, 054006 (2003)

7. E.S. Swanson, Phys. Lett. B 588, 189 (2004)

8. D. Gamermann, E. Oset, D. Strottman, M.J. Vincente Vagas, Phys. Rev. D 76, 074016 (2007)

9. J. Hoffman, M.F.M. Lutz, Nucl. Phys. A 733, 142 (2004)

10. F.K. Guo, P.N. Shen, H.C. Chiang, Phys. Lett. B 647, 133 (2007)

11. L. Maiani, F. Piccinini, A.D. Polosa, V. Riguer, Phys. Rev. D 71, $014028(2005)$

12. Y.Q. Chen, X.Q. Li, Phys. Rev. Lett. 93, 232001 (2004)

13. B.A. Li, Phys. Lett. B 605, 306 (2005)

14. M.F.M. Lutz, E.E. Kolomeitsev, Nucl. Phys. A 730, 110 (2004)

15. T. Mizutani, A. Ramos, Phys. Rev. C 74, 065201 (2006)

16. J. Hoffman, M.F.M. Lutz, Nucl. Phys. A 763, 90 (2005)

17. J. Hoffman, M.F.M. Lutz, Nucl. Phys. A 776, 17 (2006)

18. I.I. Balitsky, V.M. Braun, Nucl. Phys. B 311, 541 (1989)

19. A.K. Agamaliev, T.M. Aliev, M. Savcı, Nucl. Phys. A 958, 38 (2017)

20. T.M. Aliev, T. Barakat, M. Savci, Phys. Rev. D 93, 056007 (2016)

21. E. Bagan, M. Chabab, S. Narison, Phys. Lett. B 278, 369 (1992)

22. I.I. Balitsky, V.M. Braun, A.V. Kolesnichenko, Nucl. Phys. B 312, 509 (1989)

23. P. Ball, V.M. Braun, N. Kivel, Nucl. Phys. B 649, 263 (2003)

24. T.M. Aliev, K. Azizi, M. Savcı, Phys. Rev. D 89, 053005 (2014)

25. T.M. Aliev, A. Özpineci, M. Savcı, Phys. Rev. D 65, 096004 (2002)

26. T.M. Aliev, K. Azizi, T. Barakat, M. Savc1, Phys. Rev. D 92, 036004 (2015)

27. V.M. Belyaev, V.M. Braun, A. Khodjamirian, R. Ruckl, Phys. Rev. D 51, 6177 (1995)

28. M. Tanabashi et al., (Particle Data Group). Phys. Rev. D 98, 030001 (2018)

29. K.G. Chetyrkin, J.H. Kuhn, A. Maier, P. Maierhofer, P. Marquard, M. Steinhauser, C. Sturm, Phys. Rev. D 80, 074010 (2009)

30. A. Khodjamirian, Ch. Klein, Th Mannel, N. Offen, Phys. Rev. D 80, 114005 (2009)

31. G. Duplanic, A. Khodjamirian, Th Mannel, B. Melic, N. Offen, JHEP 0804, 014 (2008)

32. V.M. Belyaev, B.I. Ioffe, Sov. Phys. JETP 57, 716 (1983)

33. R. Rohrwild, JHEP 09, 073 (2007)

34. D. Gamermann, C.E. Jimenez-Tajero, A. Ramos, Phys. Rev. D 83, 074018 (2011)

35. M.A. Ivanov, J.G. Korner, V.E. Lyubovitskij, A.G. Rusetsky, Phys. Rev. D 60, 094002 (1999) 
36. S.L. Zhu, Phys. Rev. D 61, 114019 (2000)

37. S. Tawfiq, J.G. Korna, P.J. O’Donnels, Phys. Rev. D 63, 034005 (2001)
38. C.K. Chow, Phys. Rev. D 54, 3374 (1996)

39. K.L. Wang, X.H. Zhong, Q. Zhao, Phys. Rev. D 96, 116016 (2017) 